BNL 65225

\title{
RHIC Progress Report
}

\author{
S. Peggs
}

June 1998

\section{RHIC Project}

Brookhaven National Laboratory

Operated by

Brookhaven Science Associates

Upton, NY 11973

Under Contract with the United States Department of Energy

Contract Number DE-AC02-98CH10886 


\section{DISCLAIMER}

This report was prepared as an account of work sponsored by an agency of the United States Government. Neither the United States Government nor any agency thereof, nor any of their employees, nor any of their contractors, subcontractors or their employees, makes any warranty, express or implied, or assumes any legal liability or responsibility for the accuracy, completeness, or any third party's use or the results of such use of any information, apparatus, product, or process disclosed, or represents that its use would not infringe privately owned rights. Reference herein to any specific commercial product, process, or service by trade name, trademark, manufacturer, or otherwise, does not necessary constitute or imply its endorsement, recommendation, or favoring by the United States Government or any agency thereof or its contractors or subcontractors. The views and opinions of authors expresses herein do not necessarily state to reflect those of the United States Government or any agency thereof. 


\title{
RHIC Progress Report
}

\author{
S. Peggs, \\ Brookhaven National Laboratory, Upton, NY 11973, USA
}

\section{Abstract}

The design and construction status of the Relativistic Heavy Ion Collider, RHIC, which is in the eighth year of a nine year construction cycle, is discussed [1]. Those performance features of a heavy ion collider that are distinct from hadron colliders in general are noted. These features are derived from the experimental requirements of operation with a variety of ion species over a wide energy range, including collisions between ions of unequal energies, between protons and ions, and between polarized protons. Section 1 gives a brief introduction to the major parameters and overall layout of RHIC. A review of the superconducting magnet program is given in Section 2. Machine performance is reviewed in Section 3, and the plans for RHIC commissioning in 1999 are presented in Section 4.

\section{INTRODUCTION}

The primary motivation for colliding heavy ions at ultrarelativistic energies is the belief that it is possible to create macroscopic volumes of nuclear matter at such extreme conditions of temperature and energy density that a phase transition will occur from hadronic matter to a confined plasma of quarks and gluons. RHIC will provide head-on collisions at energies up to $100 \mathrm{GeV} / \mathrm{u}$ per beam for very heavy ions, nominally gold $\left({ }^{197} \mathrm{Au}^{79+}\right)$. The experimental program also calls for lighter ions all the way down to protons, including polarized protons. Luminosity require-

\begin{tabular}{||lrl||}
\hline \hline Kinetic energy range, Au & $10.8-100$ & $\mathrm{GeV} / \mathrm{u}$ \\
Kinetic energy range, $\mathrm{p}$ & $28.3-250$ & $\mathrm{GeV}$ \\
Number of bunches per ring & 60 & \\
Circumference & 3833.845 & $\mathrm{~m}$ \\
Number of crossing points & 6 & \\
$\beta^{*}$, injection, $\mathrm{H} / \mathrm{V}$ & 10 & $\mathrm{~m}$ \\
$\beta^{*}$, low- $\beta$ insertion, H/V & 2 & $\mathrm{~m}$ \\
Betatron tunes, H/V & $28.19 / 29.18$ & \\
Transition energy, $\gamma_{T}$ & 22.89 & \\
Magnetic rigidity, injection & 97.5 & $\mathrm{Tm}$ \\
Magnetic rigidity, top energy & 839.5 & $\mathrm{Tm}$ \\
Bending radius, arc dipole & 242.781 & $\mathrm{~m}$ \\
Maximum arc dipole field & 3.45 & $\mathrm{~T}$ \\
Maximum arc quad gradient & 71.2 & $\mathrm{~T} / \mathrm{m}$ \\
Arc magnet coil ID & 80 & $\mathrm{~mm}$ \\
Triplet magnet coil ID & 130 & $\mathrm{~mm}$ \\
& & \\
\hline \hline
\end{tabular}

Table 1: Major Parameters for the Collider.

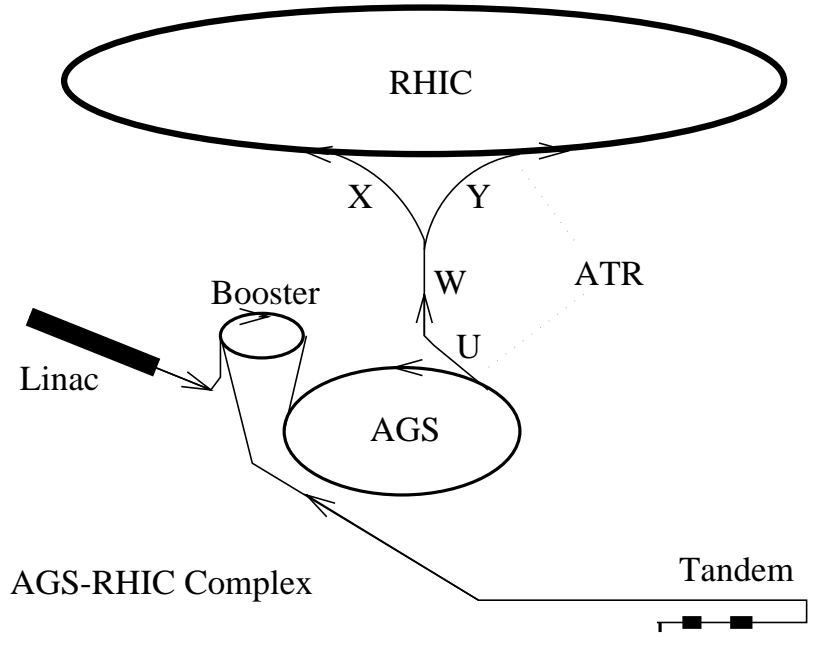

Figure 1: Schematic layout of the AGS-RHIC complex.

ments for the heaviest ions are in the $10^{26}-10^{27} \mathrm{~cm}^{-2} \mathrm{~s}^{-1}$ range. Although these luminosities are several orders of magnitude lower than p-p colliders, the higher Au-Au total cross-section results in comparable interaction rates.

The most influential experimental requirement is the need for collisions of different ion species (most notably $\mathrm{p}-\mathrm{Au}$ ) at identical Lorentz $\gamma$ 's. This necessitates accommodating mass-to-charge ratios $(A / Z)$ in the range from 1 (p) to $2.5(\mathrm{Au})$. Stabilizing the collision point location involves equalizing the rotation frequencies of the two beams, which requires the two rings to operate at different magnetic fields. The need for such beams to pass through common magnets in the interaction region (IR) dictates a lattice design different from conventional hadron colliders. Beams with large transverse and longitudinal sizes are a natural consequence of collider operations at relatively low energies, with enhanced intra beam scattering (IBS), which scales as $Z^{4} / A^{2}$. This in turn has ramifications for the lattice (strong focusing short cells of $29.6 \mathrm{~m}$ length) and for the magnet aperture ( $80 \mathrm{~mm}$ in the arcs). The RF system requirements are also determined by these considerations, and by the desire for a short interaction diamond length $(<0.2 \mathrm{~m} \mathrm{rms})$ for optimum detector design. Colliders, unlike fixed target machines, are designed to operate for extended periods at high energies. The economics of power consumption argue strongly for superconducting magnets. RHIC is such a superconducting machine.

RHIC machine parameters derived from these general requirements are outlined in Table 1 , and the layout of RHIC and its injector chain are shown schematically in Fig. 1. 


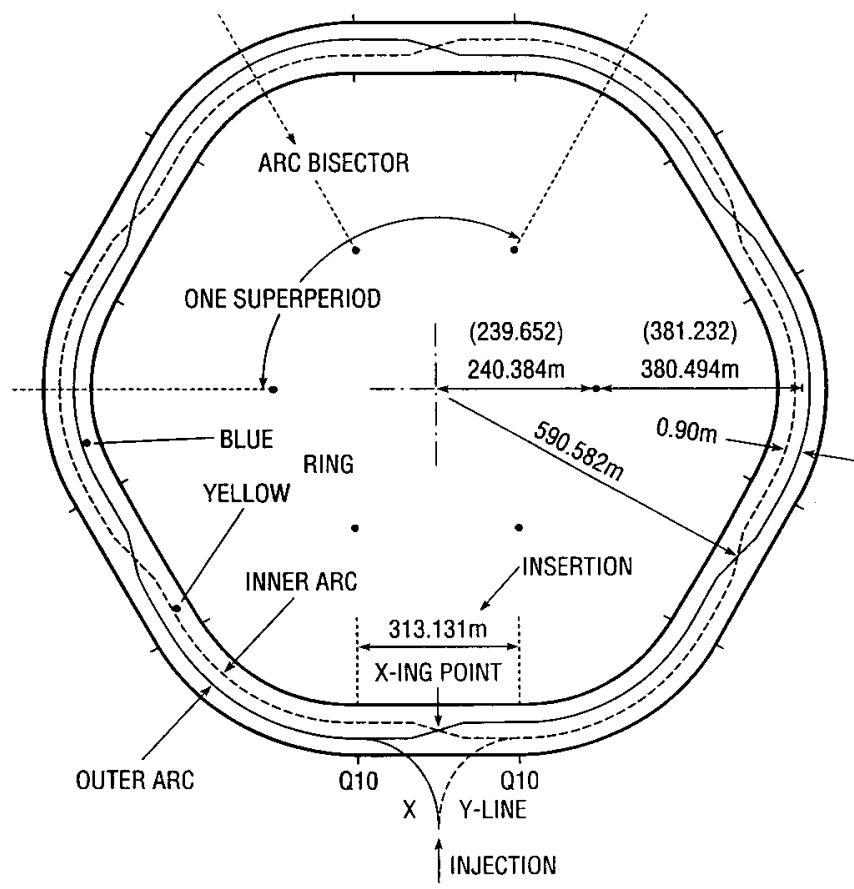

Figure 2: Layout of the RHIC collider and the tunnel.

\subsection{Layout}

The internal collider layout is shown schematically in Fig. 2. RHIC consists of two identical, quasi-circular rings separated horizontally by $0.90 \mathrm{~m}$, and oriented to intersect one another at six locations. Each ring consists of three inner and three outer arcs, separated by six insertion regions. Each arc consists of 11 FODO cells, with each half cell consisting of a single dipole and a CQS assembly containing a quadrupole, sextupole, and concentric correction elements. Beam collisions occur at a crossing point in each IR. These IRs contain the optics necessary for producing small collision betatron functions $\beta^{*}$ and a zero dispersion at the crossing point, in addition to bending the beams into head-on collisions. The focusing is relaxed at injection

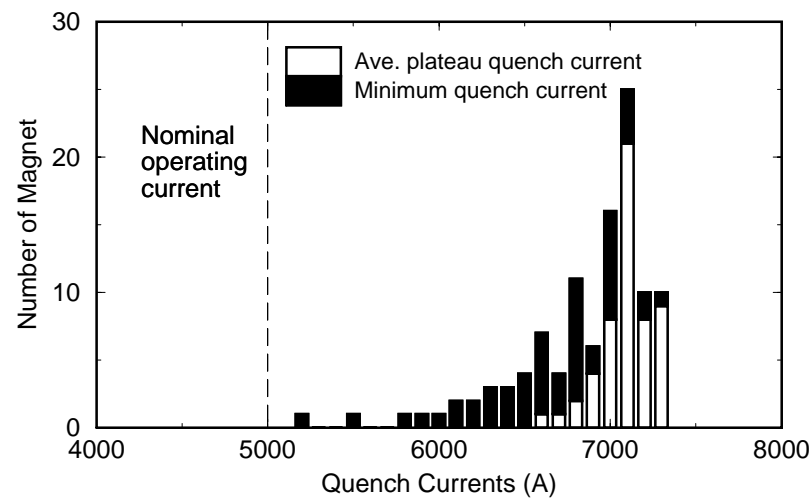

Figure 3: Arc dipole quench current distribution. with $\beta^{*}=10 \mathrm{~m}$, but during collisions at top energy it is squeezed down to a value of $2 \mathrm{~m}$, resulting in a maximum $\beta$ of about $700 \mathrm{~m}$ in the triplet quadrupoles. The warm parts of each IR contain machine utilities such as injection, beam abort, RF stations, collimators, and specialized instrumentation.

\section{MAGNETS}

RHIC magnets have a "cold-iron" design with single layer coils supported by plastic pole spacers. There is no stainless steel collar, and the cryogenic transfer lines are located in the cryostat. The arc dipoles and quadrupoles have a coil inner diameter (i.d.) of $80 \mathrm{~mm}$, and a nominal maximum field and gradient of $3.45 \mathrm{~T}$ and $72 \mathrm{~T} / \mathrm{m}$, respectively. The industrial production of $80 \mathrm{~mm}$ arc magnets was completed in a two year period from 1994 to 1996, and the BNL assembly of CQS cryogenic modules was completed in 1997. Arc dipoles were produced by the Northrop-Grumman Corporation in a build-to-print contract, as complete cryogenic elements ready for immediate installation.

Field quality and quench threshold are both crucial aspects of superconducting magnet performance. Arc dipole statistics on minimum and plateau quench currents for a set
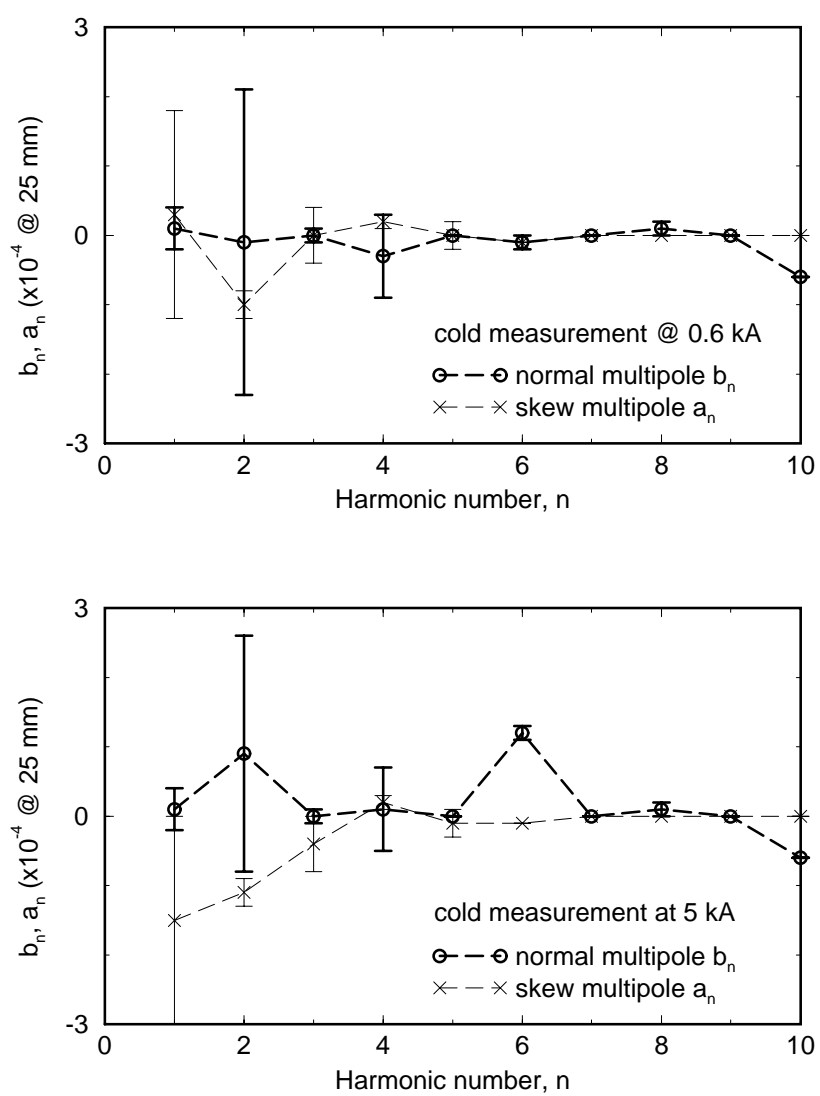

Figure 4: Arc dipole magnetic field multipole harmonics at injection $(0.6 \mathrm{kA})$ and storage $(5 \mathrm{kA})$ currents. Harmonic $n=1$ is quadrupole. 
of 60 magnets are shown in Fig. 3. The limited cold testing of only $20 \%$ of the magnets is justified by a healthy $30 \%$ quench current operating margin. None of the magnets had an initial quench current less than the nominal operating level. Limited cold testing is also justified by the good correlation between warm and cold magnetic field quality measurements, confirmed by a careful analysis of the complete data set. All of the smaller number of IR magnets (which have a smaller quench margin) are cold tested in a vertical dewar. The distribution of field harmonics in arc dipoles shown in Fig. 4 demonstrates excellent field quality with very small random multipole field components, by virtue of tight mechanical tolerances on the cable dimensions and positions. The systematic components are minimized for low-field performance at injection. Yoke saturation is apparent in the allowed harmonics at high field.

The Integral Transfer Function (ITF) of the arc dipoles was measured with beam during the Sextant Test, which was a full systems and beam test that occured from December 1996 through February 1997. Gold beam from the AGS was successfully passed through a single sextant in its final configuration, including injection kickers, RF cavities, et cetera. The average arc dipole ITF was found to differ from the test stand measurements by only about $0.2 \%$. Multiple measurements of the phase advance per FODO cell were made with different QF and QD current settings, with the results shown in Fig. 5. The beam-based arc quadrupole ITF agrees with test bench measurements within the $0.6 \%$ accuracy of the beam measurements [2].

Interaction region triplet quads have a coil i.d. of $130 \mathrm{~mm}$, with a maximum focusing strength of about $48 \mathrm{~T} / \mathrm{m}$. The dynamic aperture is determined by the triplet quadrupoles during collisions. For example, a maximum $\beta$ of $1400 \mathrm{~m}$ would occur in the triplet under an upgrade scenario with $\beta^{*}=1 \mathrm{~m}$. Under these conditions, the $5 \sigma$ beam size is expected to increase from $35 \%$ to about $70 \%$ of the triplet magnet coil radius, due to strong IBS growth. Multiple sophisticated compensation techniques are used to optimize the field quality in triplet quadrupoles, including individual error correction with tuning shims, body-end har-

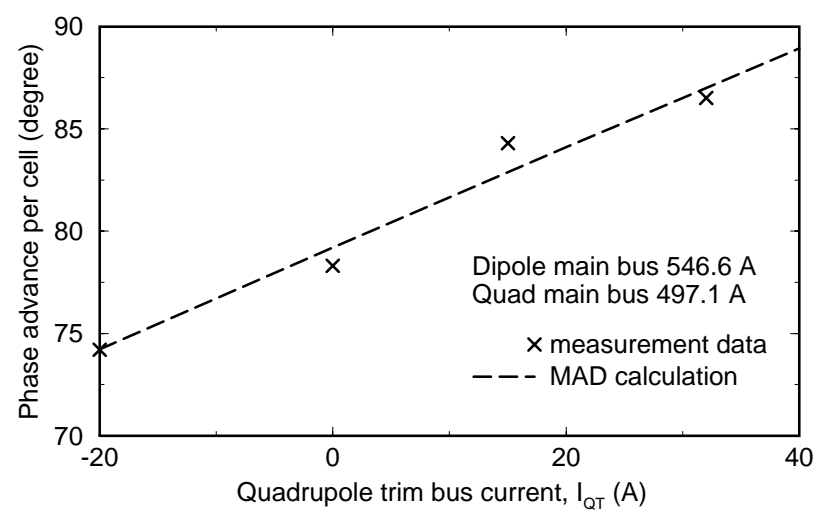

Figure 5: Measured and predicted phase advance per cell.

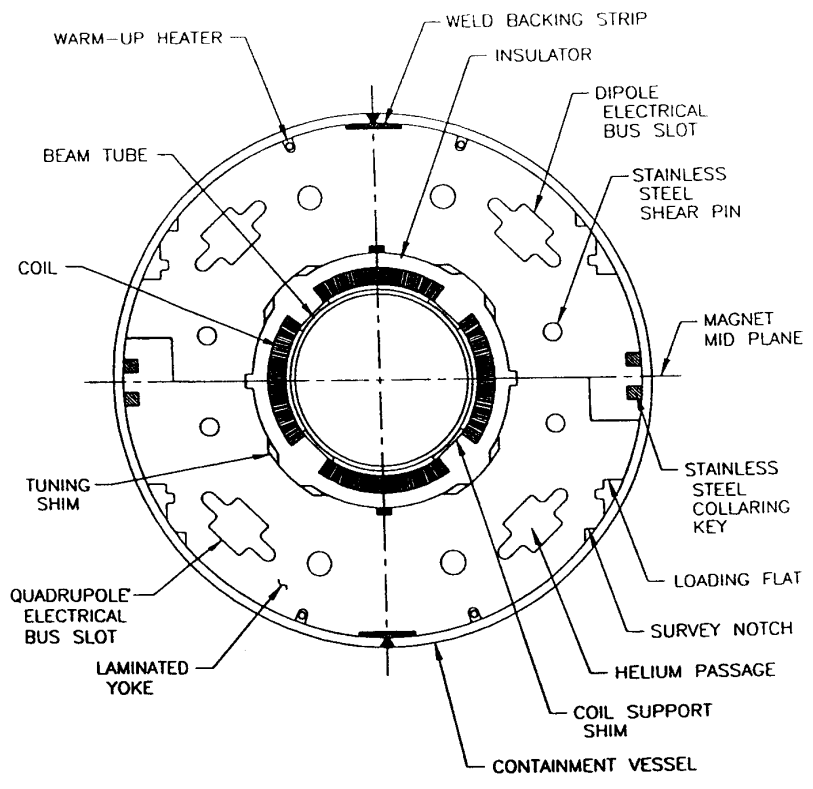

Figure 6: Triplet quadrupole cross-section showing empty slots for tuning shims.

monic compensation, magnet sorting, and lumped triplet multi-layer corrector packages. Especially tight alignment accuracies are required on these magnets. Tuning shims are inserted into the eight empty slots of the IR quadrupole body, as shown in Fig. 6, after the magnet is constructed and individually warm measured, in order to correct eight leading field harmonics. Experiments indicate that these multipole errors can be reduced to about $10 \%$ rms of their uncorrected values. The expected values for the mean and its uncertainty often become zero, while the final standard deviation is associated with a roll up of measurement errors, thermal cycling fluctuations, and quench fluctuations.

The production and assembly of most IR magnets at

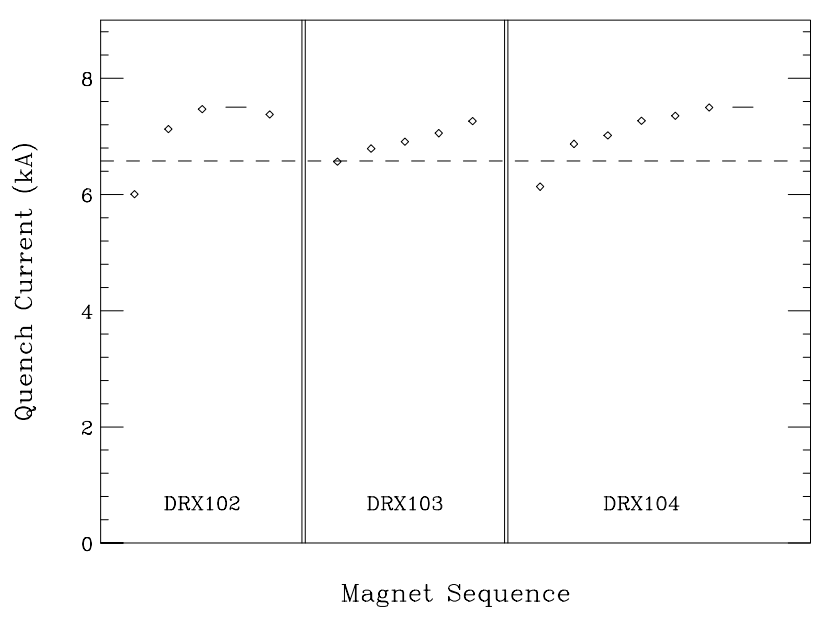

Figure 7: Training curves for the first three DX beam splitting magnets. 
BNL - correctors, triplet quadrupoles, and beam splitting dipoles - is essentially complete. The main exception is the production line for the technically challenging DX beam splitting dipoles, which have a field of 4.27 Tesla and an unusually large coil i.d. of $180 \mathrm{~mm}$. Figure 7 shows the excellent training performance of the first three production magnets. Also still in production at BNL are the helical dipoles which will be used in Siberian snakes and spin rotators during polarized proton operations.

\section{BEAM DYNAMICS}

Experience during and after the Sextant Test showed that gold beam bunch rotations are needed in the AGS before RHIC injection, raising the nominal initial longitudinal bunch area from $0.3 \mathrm{eV} \cdot \mathrm{s} / \mathrm{u}$ to $0.5 \mathrm{eV} \cdot \mathrm{s} / \mathrm{u}$. Figure 8 (top) shows the effect that IBS has on the first gold bunch injected, after it has been stored for 2 minutes while the remaining bunches are injected into both rings [3]. Although the bunch area increases by about $6 \%$ (with a nominal RF voltage of $600 \mathrm{kV}$ ), the effect is not as pronounced as with an initial bunch area of $0.3 \mathrm{eV} \cdot \mathrm{s} / \mathrm{u}$. More important is the re-bucketing gymnastic, in which beam is transferred at top
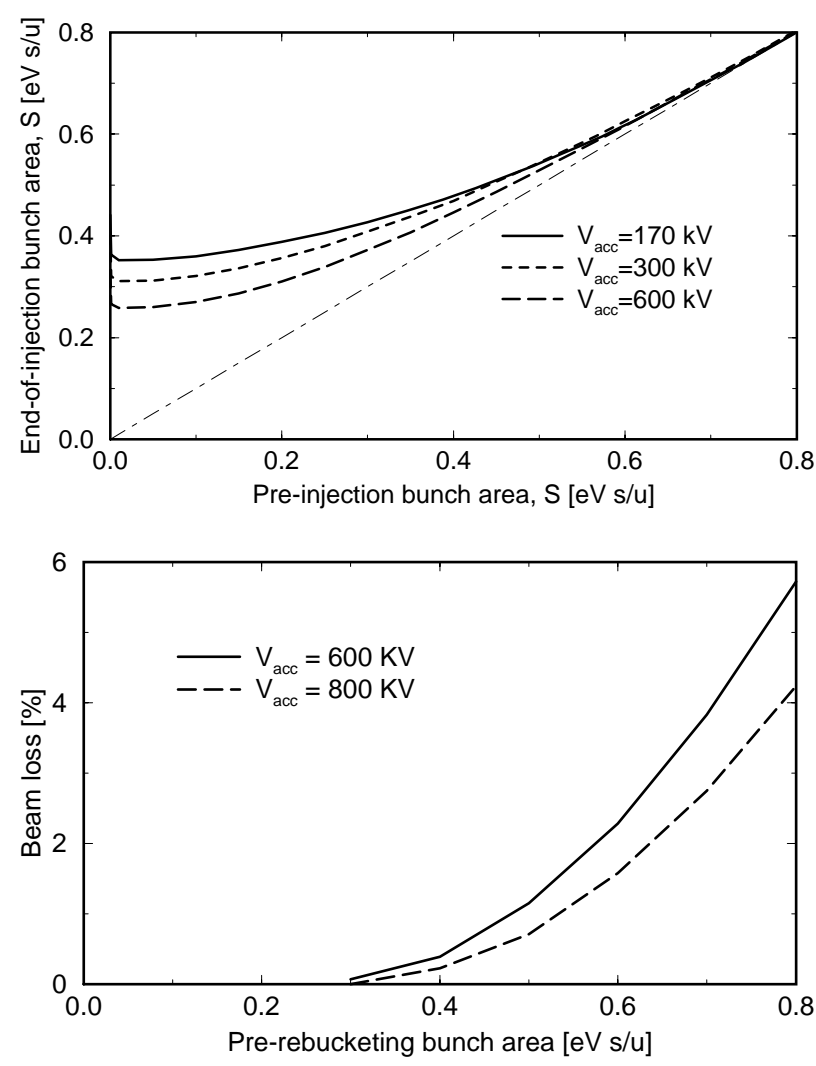

Figure 8: (Top) Growth in longitudinal area of a gold beam due to intra-beam scattering during the two minute injection period. (Bottom) Beam loss during re-bucketing as a function of initial bunch area, for nominal $(600 \mathrm{kV})$ and potential $(800 \mathrm{kV})$ excitations of the $28 \mathrm{MHz}$ RF system.

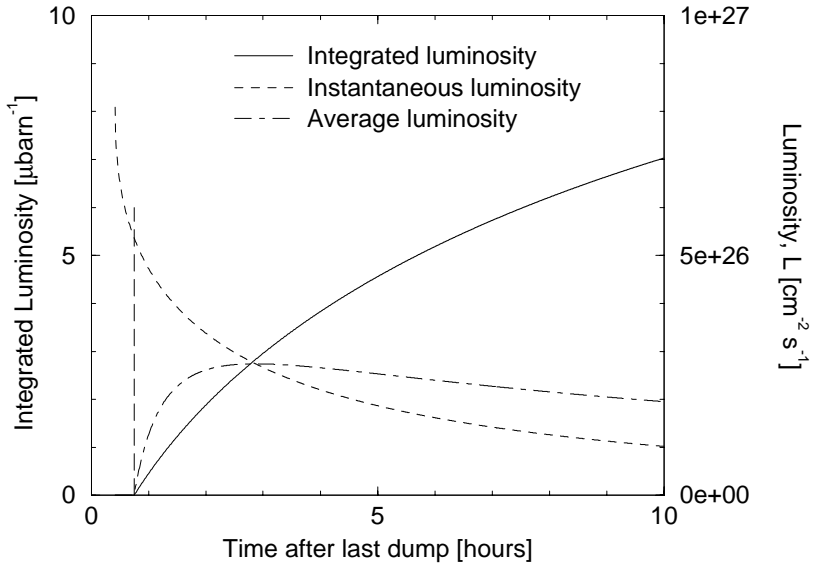

Figure 9: Integrated, instantaneous, and average luminosities during a nominal store of gold ions.

energy from the $28 \mathrm{MHz}$ RF system used for acceleration to the $197 \mathrm{MHz}$ system used during storage. Figure 8 (bottom) indicates that $2 \%$ to $3 \%$ of the beam will be lost from the storage buckets (but not from the machine). Transition crossing is not expected to play a major role in beam losses or bunch area growth [3]. Beam continues to spill over the edge of the RF buckets during storage with nominal intensities, and the transverse emittances continue to grow, with a characteristic time of order one hour. The transverse emittance will be monitored by ionization profile monitors with single turn capabilities, and possibly by a infrared detector array observing synchrotron light generated at the edge of the DX magnets [4]. Beam halo will be scraped by a collimation system which incorporates a novel crystal "pre-collimator", approximately 10 meters upstream of the primary collimator [5].

Figure 9 shows the expected "shot profile" performance with the beam parameters quoted in Table 2, using a beam dynamics model which, in addition to IBS effects, includes beam losses to the dynamic aperture and due to luminosity. Emittance growth and beam loss lead to a factor of 8 loss in instantaneous luminosity during a nominal 10 hour store. Approximately $40 \%$ of the bunch intensity is lost, mostly through IBS, but also with a significant contribution from Coulomb interactions. Gold on gold cross sections at 100 $\mathrm{GeV} / \mathrm{u}$ include 117 barns for electron pair production and capture, and 95 barns for Coulomb nuclear dissociation. If 2 experiments experience an instantaneous luminosity of $L=8 \times 10^{26} \mathrm{~cm}^{-2} \mathrm{~s}^{-1}$, the partial current lifetime due to nuclear interactions is approximately 49 hours.

\section{COMMISSIONING}

Full beam commissioning is scheduled to begin in March 1999 in a "Test" run that will begin as soon as full cool down has been completed, and the final beam pipe has been installed. First circulating beam is expected in this run. A spring shutdown for final collider installation of collimators 


\begin{tabular}{||llll||}
\hline \hline Quantity & Injection & $\begin{array}{l}\text { Store } \\
\text { (begin) }\end{array}$ & $\begin{array}{l}\text { Store } \\
\text { (end) }\end{array}$ \\
number of bunches & 60 & 60 & 60 \\
collision $\beta^{*}[\mathrm{~m}]$ & 10.0 & 2.0 & 2.0 \\
& & & \\
GOLD & & & \\
kinetic energy $[\mathrm{GeV} / \mathrm{u}]$ & 10.4 & 100 & 100 \\
ions per bunch & $10^{9}$ & $10^{9}$ & $6 \times 10^{8}$ \\
$95 \%$ bunch area $[\mathrm{eV} \cdot \mathrm{s} / \mathrm{u}]$ & $0.2-0.5$ & $0.3-0.7$ & 1.1 \\
$95 \%$ emittance $[\mu \mathrm{m}]$ & 10 & 15 & 43 \\
rms bunch length $[\mathrm{m}]$ & $0.55-0.88$ & $0.11-0.17$ & 0.22 \\
$\mathcal{L}\left[10^{26} \mathrm{~cm}^{-2} \mathrm{~s}^{-1}\right]$ & & 8 & 0.9 \\
& & & \\
PROTONS & & & \\
kinetic energy $[\mathrm{GeV}]$ & 28.3 & 250.7 & 250.7 \\
ions per bunch & $10^{11}$ & $10^{11}$ & $10^{11}$ \\
$95 \%$ bunch area $[\mathrm{eV} \cdot \mathrm{s}]$ & 0.5 & 0.5 & 1.2 \\
$95 \%$ emittance $[\mu \mathrm{m}]$ & 20 & 20 & 29 \\
rms bunch length $[\mathrm{m}]$ & 0.51 & 0.09 & 0.14 \\
$\mathcal{L}\left[10^{31} \mathrm{~cm}^{-2} \mathrm{~s}^{-1}\right]$ & & 1.5 & 1.1 \\
\hline \hline
\end{tabular}

Table 2: Evolution of RHIC parameters during a 10 hour store, mainly due to intra-beam scattering.

and for detector roll-in, et cetera, will be followed by a "Commissioning" run. First collisions and RHIC project completion are nominally expected to occur about half way through this run. Production running for the experiments, with gold ions, is scheduled to begin in fall 1999, and will last 37 weeks. Spin physics with polarized protons may start in 2000.

Goals for the end of the "Year One" production run include $50 \%$ uptime, a store set up time of less than 2 hours, and a luminosity of $10 \%$ of the design. This will require aggressive development of the luminosity parameters listed in Table 2, in particular the large number of bunches, and low values of $\beta^{*}$ with well tuned linear optics [6]. The Sextant Test experience of early 1997 showed that there is good reason to believe that it will be relatively easy to achieve the design value of the gold bunch intensity.

\section{CONCLUSIONS}

RHIC has one year of its nine year construction cycle to go before heavy ion operations begin in 1999. Industrial production of superconducting magnets has been completed, while in house production of specialized magnets is essentially complete, except for the DX beam splitting dipoles. The major milestone of the Sextant Test was passed in early 1997 , successfully commissioning many of the beam and non-beam systems. Intra-beam scattering is expected to be a dominant physical phenomenon limiting the ultimate performance of RHIC in heavy ion operations. Polarized proton operations are scheduled to begin in 2000 .

\section{REFERENCES}

[1] "RHIC Design Manual", www.rhichome.bnl.gov/NTshare/rhicdm .

[2] D. Trbojevic et al, "Results from Betatron Phase Measurements in RHIC during the Sextant Test", these proceedings.

[3] J. Wei et al, "RHIC Longitudinal Parameter Revision", these proceedings.

[4] D. Trbojevic, E. Courant, and S. Peggs, "A Possible Synchrotron Light Beam Profile Monitor in RHIC", these proceedings.

[5] D. Trbojevic et al, "A Study of RHIC Crystal Collimation", these proceedings.

[6] D. Trbojevic et al, "Measurements of the Betatron Functions and Phases in RHIC", these proceedings. 